\title{
The Mars Global Surveyor Mission: Description, Status, and Significant Results
}

\author{
Arden Albee \\ Division of Geological and Planetary Sciences, California Institute of \\ Technology, Pasadena CA 91125 USA
}

\begin{abstract}
The Mars Global Surveyor (MGS) spacecraft entered an elliptical orbit at Mars on September 11, 1997. Until March 1999 it acquired scientific data from decreasing-sized orbits as it alternated between aerobraking and nadir-pointing modes. This time period provided tremendous advances in our knowledge of the shape and topography, the gravity field, the magnetic field, and the atmospheric structure and dynamics of Mars. In April 1999 MGS entered its planned two years in the mapping mode. In this mode the high-gain antenna tracks Earth so that the instruments can take data continuously and so that the camera system can return high-resolution data in real-time. IR spectral and temperature data, as well as high-resolution images are providing new insight into the geologic evolution of Mars. All data is being archived at about six month centers so that it is available in electronic format to the international community.
\end{abstract}

\section{Introduction}

Mars Global Surveyor (MGS) is the initial mission in the NASA Mars Surveyor Program, an integrated series of missions to explore Mars. Over the next decade the Program will launch orbiters or landers every 25 months, using advanced technology to develop a comprehensive portrait of Mars. The Program focuses on understanding present and past climatic conditions on Mars, determining whether Mars developped prebiotic compounds and life and identifying resources that might be of use during human expeditions to the surface. Determining the locations and states of water reservoirs now and in the past are key objectives.

\section{Description of the mission}

The objective of Mars Global Surveyor is to make a variety of observations with global coverage over a complete Mars year, studying the atmosphere, the surface and the interior. Studies had shown that this could be done economically by a spacecraft in a nearly polar orbit, carrying a suite of complementary instruments that would operate concurrently and continuously. In order to reduce launch mass the mission plan replaced propulsive maneuvers by about four months of aerobraking. This process utilizes repeated dips into the upper atmosphere to slow the spacecraft down from its initial 45 hour elliptical orbit to the final two hour near-circular mapping orbit. Large solar array panels extend from each 
side of the spacecraft and provide the aerobraking drag. The mapping orbit is near circular, near polar, and sun-synchronous and has a 117-minute period with a 7-day near-repeat cycle. As a result the planet is repeatedly mapped in 26day cycles with a constant Sun angle ( 2 a.m., 2 p.m.), permitting differentiation between those characteristics that vary daily from those that are due to seasonal changes. No movable scan platform is provided as in most past missions. During the mapping configuration at Mars the spacecraft is continuously nadir-pointed, rotating at the orbital rate, as the antenna tracks Earth and the solar arrays track the Sun. Measurements are made continuously from the mapping orbit over the 687-day Martian year, permitting repetitive observations of the surface and gravity field and seasonal variations of the atmosphere and magnetic field.

The experiments include: Mars Orbiter Camera (MOC), a system of three line-scan cameras; TES, a Thermal Emission Spectrometer; Mars Orbiter Laser Altimeter (MOLA), a laser altimeter; Radio Science (RS), using the spacecraft radio system as controlled by an ultrastable oscillator; MAG-ER, dual MAGnetometers complemented with an Electron Reflectometer; and Mars Relay (MR), a radio communication system to relay data to Earth from landers on the surface of Mars. Each of the instruments and the purpose of the experiment are described in separate papers in a special issue (1992, JGR, 97, 7663-7814). The spacecraft accelerometer and the horizon sensor were utilized as additional atmospheric sensors during the aerobraking period.

Table 1. Home Sites for MGS and the Instruments

\begin{tabular}{ll}
\hline MGS & http://mars.jpl.nasa.gov/mgs/index.html \\
MAG/ER & http://mgs-mager.gsfc.nasa.gov/ \\
MOC & http://www.msss.com/ \\
MOLA & http://ltpwww.gsfc.nasa.gov/tharsis/mola.html \\
RS & http://nova.stanford.edu/projects/mgs/dmwr.html \\
TES & http://emma.la.asu.edu/ \\
\hline \hline
\end{tabular}

\section{Mission overview}

The MGS spacecraft was launched on November 7, 1996. After orbital insertion into a 45-hour elliptical orbit at Mars on September 11, 1997 MGS entered a period of aerobraking. The spacecraft utilized repeated dips into the upper atmosphere to slow it so as to attain the low altitude $(378 \mathrm{~km})$ circular-mapping orbit. According to plan it would have reached this orbit early in spring of 1998 . However, deployment in early cruise resulted in damage to a solar panel, the extent of which did not become clear until about a month into aerobraking. As a result the orbit was raised higher in the atmosphere to lower the pressure on the panel and the science instruments were operated in their preferred nadir orientation in the near-periapsis portion of each 35.4 hour elliptical orbit for a month during assessment of the problem. In these 16 orbits the periapsis altitude was only $174 \mathrm{~km}$ and the spacecraft passed in and out of the ionosphere on each orbit. Excellent, although unanticipated, science data was acquired during this period as alternative plans were considered (1998, Science, 279, 1671- 
1697). Entry into the circular mapping orbit was delayed for an entire Martian year, requiring many more lower-pressure aerobraking dips into the atmosphere. The 378-km circular orbit was reached on February 19, 1999, but in the 2:00 a.m. (relative to the Sun) position rather than in the originally planned 2:00 p.m. position. This delay made it possible to operate the spacecraft and its instruments in the geometric relationships for which they had been designed.

Scientific observations were obtained during most of this delay. Low-altitude (high-resolution) data, especially important for the magnetometer and gravity investigations, were obtained over most of the planet. Such coverage was possible because during this period of time the periapsis position of the continuouslydecreasing elliptical orbit migrated from $45^{\circ}$ North over the north pole and then down and over the south pole. Correct phasing of the entry into the circular orbit necessitated a pause in the aerobraking operation, during which science data was collected from 372 elliptical orbits, $175 \mathrm{~km}$ by $17,850 \mathrm{~km}$, from March 27, 1998 to September 23, 1998. During the periapsis portion of each 11.6hour orbit the spacecraft was turned to the nadir-pointing position so that the instruments pointed to Mars for about 22 minutes. The altimeter, thermal emission spectrometer, and camera obtained data in near-normal mode during the nadir portion of these orbits and acquired lower-resolution global image and thermal data during the rollout from the nadir position to the earth-point position. The magnetometer-electron reflectometer obtained data throughout the entire orbit during most of this period, but radio tracking was limited.

Science data was also obtained during the aerobraking orbits. The atmospheric density of Mars at the aerobraking altitude demonstrated great variation over time as well as large orbit-to-orbit differences. So that the spacecraft could adjust its orbit, the density had to be predicted for each orbit to determine the appropriate and safe depth within the atmosphere for the aerobraking passage. Although science data acquisition during the aerobraking phase was not in the original mission plan, MOC, TES, the accelerometer, the electron reflectometer, and the horizon sensor all acquired data to support prediction of the atmospheric density. MGS returned 2140 MOC images, 11 million TES spectra, 206 MOLA profiles with 2.6 million points, 465 radio-occultation profiles and 1000 MAG low altitude passes during this unexpected bonus prior to the primary mission. Moreover, atmospheric data were obtained over a range of daily times, other than the fixed 2:00 am/pm position of the mapping mission.

After a period of orbit adjustment, instrument calibration, gravity measurements, and acquisition of a seven-day data set from all instruments the high-gain antenna (HGA) was deployed on its boom from the fixed position attached to the body of the spacecraft. This deployment permitted initiation of the planned mapping mission, which requires the HGA to track Earth and return data simultaneously with data taking by the instruments.

\section{Significant results}

MOC is currently photographing portions of Mars at unprecedented resolution looking at the surface effects of wind, water, ice, volcanic eruption, earthquake faulting, slope failures, and other geological processes. The narrow-angle camera can capture small areas at its highest resolution of $1.4 \mathrm{~m}$ per pixel, but because 
of the high data volume required only small "samples" - postage stamps - can be imaged at this resolution. Features as small as 3-4 m across, such as boulders, craters, and dunes, are recognizable and evidence of the action of surface processes are visible in every high resolution image. MOC also scans the entire planet daily with the red and blue wide-angle cameras in order to build up synoptic coverage of the surface and atmosphere, much like earth-orbiting weather satellites. These cameras also image targeted strips at a medium resolution and will produce color stereoimagery of the entire surface over the course of the mission.

TES uses a Michelson interferometer that measures the emitted infrared spectrum, at high spectral resolution to determine the mineral composition and thermal properties of Martian rocks and soils and the composition of ices, atmospheric dust, and clouds. TES continuously measures temperature and pressure, providing 12 profiles per day of the atmosphere from pole to pole. These provide a basis for understanding the atmospheric dynamics and the energy balance between the Martian surface and atmosphere. In addition, as the radio transmission from the spacecraft passes through the Mars atmosphere it is diffracted by the atmospheric electrons and provides high vertical resolution profiles of the temperature and pressure in the atmosphere. Preliminary analyses of the TES spectra, after removal of the atmospheric and dust components, are providing insight into the surface thermal properties (grain size) and the mineral composition of the surface materials (predominantly "basaltic").

MOLA uses the flight time of a laser pulse from the spacecraft to the surface and return in conjunction with knowledge of the spacecraft position to map the shape and topography of Mars with precision of about five meters. The laser fires ten pulses per second illuminating $\sim 160-\mathrm{m}$ circle on the surface. MOLA has now provided a precise map of the shape and topography of Mars, now known better than the Earth's continents in an overall sense. The most striking feature remains the $5 \mathrm{~km}$ difference in elevations and the difference in roughness between the smooth northern plains and the southern cratered highlands.

The RS team use data provided by the spacecraft's radio system and an onboard ultrastable oscillator to map spatial variation in the gravity field of Mars by measuring instantaneous velocity changes of the spacecraft in its orbit. Removal of the gravitational signal of the topography makes it possible to gain some understanding of the internal density anomalies that are associated with thermal or compositional differences in the interior of the planet. The rough, elevated southern hemisphere has a relatively smooth gravitational signature indicating a state of near-isostatic compensation, while the low, flat northern plains display a wider range of uncompensated gravity anomalies that indicates a thinner but stronger crust than in the south.

The MAG investigation measures the magnetic field on Mars. The presence of a magnetic field provides direct evidence for the existence of active motions in a fluid core, either at the present or at some time in the past. The electron reflectometer measures electronic properties as the spacecraft passes through the upper atmosphere and can infer the presence of weak remnant crustal magnetization. Almost immediately upon arrival at Mars the magnetometer experiment showed that Mars lacks a global magnetic field at the present time. This resolved a long-standing controversy and places an important constraint on the present 
nature of the Martian core. More importantly, however, during the low portions of the elliptical orbit the magnetometer has mapped crustal magnetic anomalies of surprisingly high strength in the older rocks of the southern hemisphere and these anomalies are interpreted as evidence of a vigorously convecting dynamo in the early core.

Currently data are being returned at a very high rate and are being archived for electronic public access by the Planetary Data System (hyperlink: http://www.pds.jpl.nasa.gov). Published collections of early results from Mars Global Surveyor are: 1998, Science, 279, 1597-1816; 1998, GRL, 25, 4393-4420; 1999, Science, 284, 1495-1502; 1999, Nature, 397, 584-594, 2001, JGR, in press.

Table 2. Significant Achievements of MGS at Mars During the First Mapping Year

1 Significant remnant magnetization of the Martian crust is evidence of an early molten interior with a vigorous core dynamo. No current global field (MAG).

2 Reliable global models of the structure of the crust and lithosphere show near isostatic compensation in the rougher south and a range of uncompensated gravity anomalies in the smoother north (RS \& MOLA).

3 The best global topographic model produced for any planet, including Earth, shows a $30-\mathrm{km}$ range of topography, a pole-to-pole slope that controlled the transport of water in early Martian history, and a flat northern depression that may represent the location of a large ancient ocean (MOLA).

4 Thick layered sequences of strata in Valles Marineris suggest the possibility of extensive volcanism during early to mid-Martian history (MOC).

5 Thermal emission spectra show wide occurrence of basaltic rocks in the south and andesitic rocks in the north. Widespread occurrence of plagioclase and pyroxene and the lack of weathered hydrated mineral attest to the absence of pervasive weathering. High-albedo areas have a non-diagnostic spectrum, much like the atmospheric dust (TES).

6 Detection of several coarse-grained hematite deposits, possibly indicative of deposition in a surface hydrothermal environment. No areas of carbonate, sulfate, or quartz have been detected (TES).

$7 \quad$ Evidence for a complex depositional and erosional history elucidates the importance of aeolian transport on the global and local scale (MOC).

8 A reliable estimate of water volume in the present polar caps and evidence for distinctive evolution of the north and south polar caps (MOLA \& MOC).

9 Clear evidence of a sapping origin of many channels from probable melting of ground ice and possible evidence for recent liquid water in numerous spatially-isolated regions (MOC).

10 Significantly improved understanding of atmospheric dynamics from continued monitoring of temperature, pressure, and opacity, and measurement of cloud heights (TES, RS, MOLA, ACC, MOC). Value of data is greatly enhanced through computer simulations using general circulation models (GCMS). 